\title{
Internet Addiction
}

\author{
Abdulkareem Salman Khudhair(M.N.S)
}

College of Nursing, University of Basra Iraq

DOI: $10.36347 /$ sjmcr.2020.v08i04.025

| Received: 14.04.2020 | Accepted: 21.04.2020 | Published: 30.04.2020

*Corresponding author: Abdulkareem Salman Khudhair

\section{Abstract}

Internet addiction is a wide term that covers a range of impulse-control problems and behaviors involving the internet, mobile technology, and personal computer, while there are no officially accepted criteria yet to diagnose internet addiction. The Internet in some way has made our lives easier, for example, it provides easy access to entertainment and information. However, when used excessive amounts, it can cause, anxiety obsession, depression, and also isolation, which are all symptoms of Internet addiction. Internet addiction is listed to be yet within the latest edition of the statistical manual and diagnostic of mental disorders. However, a 2-year study funded by the National Institutes of health may change that. Beginning in August of 2017, the study could deliver sufficient evidence that problems stemming from more internet use deserve serious attention from U.S. mental health and psychiatric communities. Professionals that do recognize internet addiction tend to classify it as either an impulse control disorder or obsessivecompulsive disorder or to assist treatment. Internet addiction is additionally called internet dependence compulsive computer use, and pathological internet use.

Keyword: Internet, adduction, Net Compulsions.

Copyright @ 2020: This is an open-access article distributed under the terms of the Creative Commons Attribution license which permits unrestricted use, distribution, and reproduction in any medium for non-commercial use (NonCommercial, or CC-BY-NC) provided the original author and source are credited.

\section{TYPES OF INTERNET ADDICTION}

Researchers have identified 5 subcategories of specific types of computer and internet addictions.

\section{Cybersex Addiction}

Addiction on cybersex is one of the more selfexplanatory internet addictions. An obsession with any of those services is harmful to one's ability to create real-world intimate relationships, sexual, or romantic. Treatment options are available for those with cybersex addictions, typically within the sort of intervention followed by ongoing inpatient or outpatient therapy.

\section{Net Compulsions}

Net compulsions concern interactive activities online which will be extremely harmful, like online trading stocks, gambling, and compulsive online shopping and, online auctions. These habits can have a detrimental impact on one's disrupt job-related duties and financial stability. Spending or losing excessive amounts of cash also can also cause stress in one's relationships. With instant and straightforward access to online casinos and stores, it's easy for those that are already prone to gambling or spending addiction to urge hooked online.

\section{Cyber (Online) Relationship Addiction}

online or cyber relationship addicts are deeply involved with maintaining and finding relationships online, often forgetting and neglecting real-life family and friends. Typically, online relationships are formed in different social networking sites or chat rooms but can occur anywhere you'll interact with people online. Often, people that pursue online relationships do so while concealing their real identity and appearance, these modern phenomena led to the creation of the term "catfish."

\section{Compulsive Information Seeking}

The internet provides users with knowledge and wealth of data. For some, the chance to search out information so easily has become an uncontrollable urge to collect and organize data. Information seeking in some cases could be a manifestation of pre-existing, obsessive-compulsive tendencies. Commonly, compulsive information-seeking also can reduce work productivity and potentially result in job termination. Looking at the severity of the addiction, treatment options can range from different therapy modalities that concentrate on changing compulsive behavior and developing coping strategies to medication. 


\section{Computer or Gaming Addiction}

Addiction on the computer sometimes remarked as computer gaming addiction involves onand offline activities which will be finished a computer. As computers became more widely available, games like Minesweeper, Solitaire, Tetris, and were programmed into their software. Researchers quickly found that obsessive video game playing became an issue in certain settings. Office employees would spend excessive amounts of your time playing these games causing a notable decrease in productivity. Today, there are thousands of new ones available beside these classic games. Computer addiction is the oldest sort of internet/computer addiction, and it's still prevalent and harmful today.

\section{Causes of Internet addiction}

In addition to high exposure to technology, there are many other reasons why someone can become an online addict. For instance, people played by depression and anxiety can have the predisposition to possess an online addiction; their lack of emotional support makes them address the web to fill this need. Another example is those that are overly shy, suffer from low self-esteem, have feelings of inadequacy, fear of disapproval, and can't seem to be ready to build relationships with their peers. These people, as a consequence, find you hoping on the net for comfort and to make fake personas as a picture of who they might wish to be.

\section{What Are the Signs of an Online Addiction Problem?}

An addiction to the Internet is manifested in both physical and emotional symptoms; however, these specifics may vary for every person. These are basically warning signals that addiction could also be developing. If you are feeling that you simply or love has these symptoms, it's not yet too late. All it takes could be a telephone to and that we can facilitate you.

\section{Emotional Symptoms of Online Addiction}

The following symptoms are typical of online addicts:

- Anxiety

- Dishonesty

- Feelings of guilt

- Depression

- Isolation

- Agitation

- Euphoric feelings when in front of the computer

- Unable to keep schedules

- No sense of time

- Defensiveness

- $\quad$ Avoiding doing work

\section{Physical Symptoms of Online Addiction}

The following symptoms are characteristic of someone who uses the computer for a very long period of time:

- Backache

- Headaches

- Blurred or strained vision

- Disturbances in sleep

- Carpal tunnel syndrome

- Weight gain or loss

\section{Warning signs}

- Excessive preoccupation with the Internet.

- Use of the Internet in increasing amounts of your time so as to attain satisfaction.

- Repeated, unsuccessful efforts to manage, in the reduction of or stop Internet use.

- Feelings of restlessness, depression, irritability, or moodiness when attempting to chop down the use of the Internet Jeopardized or risked the loss of great relationships, job, educational or career opportunities due to Internet use.

- Lies to relation, therapists, or others to hide the extent of involvement with the Internet.

- The use of the net may be thanks to escaping from problems or feelings of hopelessness, depression, guilt, and anxiety.

\section{Treating an Internet Addiction}

There is no body-specific treatment that should be used to address internet addiction. Betting on the severity of the addiction and also the behaviors of the individual, differing kinds of treatment would be effective. If someone you recognize is stricken by excessive internet abuse, the primary step is to plan an intervention or to precise your concerns with their behaviors. Therapy is mostly incorporated into the treatment of addiction together with any co-occurring disorders which will be present like anxiety, depression, and/or obsessive-compulsive disorder. In some cases, medication could also be used to manage symptoms of those underlying mental illnesses or to regulate intrusive thoughts about going surfing if other treatment options weren't effective.

\section{Helping a friend or relative with a computer or internet addiction}

- Introduce the net addict to others who handle their use of the net reasonably

- Serve as an honest leader for the net addict by properly managing your own use of computers and therefore the Internet

- Support a person's desire for change if it appears that he or she is an online addict

- Talk to the person about your concerns that he or she is also an online addict

- Get the person involved in interests that aren't associated with the net 
- Encourage an online addict to hunt professional counseling

\section{REFERENCES}

1. http://www.psychguides.com/guides/computerinter net-addiction-symptoms-causes-and-effects/.

2. http://www.addictionrecov.org/Addictions/index.as $\mathrm{px}$ ?AID $=43$
3. http://news.stanford.edu/news/2006/october18/med -internet-101806.html

4. http://www.psychguides.com/guides/getting-helpfor-an-internet-addiction/

5. https://www.addictioncenter.com/drugs/internetaddiction/

6. https://www.psychguides.com/behavioraldisorders/computer-internet-addiction/ 